\title{
Effects of Triangular Core Rotation of a Hybrid Porous Core Terahertz Waveguide
}

\author{
Sharafat Ali, Nasim Ahmed, Syed Alwee, Monirul Islam, Sohel Rana, and Touhid Bhuiyan
}

\begin{abstract}
In this paper, we investigate the effects for rotating the triangular core air hole arrangements of a hybrid design porous core fiber. The triangular core has been rotated in anticlockwise direction to evaluate the impact on different waveguide properties. Effective Material Loss (EML), confinement loss, bending loss, dispersion characteristics and fraction of power flow are calculated to determine the impacts for rotating the triangular core. The porous fiber represented here has a hybrid design in the core area which includes circular rings with central triangular air hole arrangement. The cladding of the investigated fiber has a hexagonal array of air hole distribution. For optimum parameters the reported hybrid porous core fiber shows a flat EML of $\pm 0.000416 \mathrm{~cm}^{-1}$ from 1.5 to 5 terahertz (THz) range and a near zero dispersion of $0.4 \pm 0.042 \mathrm{ps} / \mathrm{THz} / \mathrm{cm}$ from 1.25 to 5.0 THz. Negligible confinement and bending losses are reported for this new type of hybrid porous core design. With improved concept of air hole distribution and exceptional waveguide properties, the reported porous core fiber can be considered as a vital forwarding step in this field of research.
\end{abstract}

Keywords-porous core waveguide, THz waveguide, flat EML, hybrid design, flat dispersion, triangular design, core rotation

\section{INTRODUCTION}

$\mathbf{T}$ ERAHERTZ (THz) frequency can be roughly defined from 0.1 to $10 \mathrm{THz}$ range of frequency lies between microwave and infrared radiation on electromagnetic spectrum [1]. This practical band is often referred as "THz gap" due to the previous difficulties to exploit this region. It resembles the transition between electrical and optical frequencies for which adapting technologies for $\mathrm{THz}$ band has been proven difficult in the past. Nowadays, $\mathrm{THz}$ frequency regime has become an emerging field of research for its potential applications such as biomedical sensing [2], spectroscopy [3], astronomy [4], monitoring drugs [5], explosives or weapons in a nondestructive manner [6], non-invasive imaging [7], hybridization of DNA [8] and communications [9]. In the Terahertz technology the three major contributing fields are terahertz source, terahertz waveguide and terahertz detector [10]. Although advanced technologies for terahertz source for

Sharafat Ali is with Center of Excellence, Advanced Communication Engineering, University Malaysia Perlis (e-mail: sharafat.ali@ieee.org ).

Nasim Ahmed is with School of Computer And Communication Engineering, University Malaysia Perlis (e-mail: nasim@theiet.org).

Syed Alwee is with School of Computer And Communication Engineering, University Malaysia Perlis . (e-mail: syedalwee@unimap.edu.my).

Monirul Islam is with University Malaysia Perlis (e-mail: s.m.1979 @ieee.org).

Sohel Rana is with Department of Electrical \& Electronic Engineering, Bangladesh University (e-mail: dsmsrana@gmail.com).

, and Touhid Bhuiyan is with Department of Software Engineering, Faculty of Science and Information Technology, Daffodil International University (email: headswe@daffodilvarsity.edu.bd). generation and terahertz detector for detection of the $\mathrm{THz}$ system were already developed during the past few decades, most of the commercially available terahertz systems are still based on free space propagation. The reason behind this is the lack of commercially feasible low loss waveguides $[11,12]$. Previously, various types of waveguides were proposed for the $\mathrm{THz}$ wave propagation, such as metallic wires [13], dielectric metal-coated tubes [14], Bragg fibres [15] all-dielectric subwavelength polymer fibres [16] etc. But still these proposed waveguides had to challenge to satisfy flexible, efficient, lowloss transmission of broadband $\mathrm{THz}$ waves for long-length delivery. In order to achieve lower the propagation loss, a large part of transmitted radiation should be confined in air while propagating small portions into the material $[17,18]$ as dry air has lower absorption loss and dispersion than any other material in the $\mathrm{THz}$ band $[10,19]$. In recent years researchers have reported porous core photonic crystal fibers (PCFs) as an improved option for $\mathrm{THz}$ wave transmission. Such porous fibers can be tuned to gain different optical characteristics by simply changing its parameters such as size and arrangement of the air holes, distance between air holes and the air hole structure.

Several porous core fibers designs have been reported for $\mathrm{THz}$ transmission in the resent years. J. Liang et al.[20] had proposed a $\mathrm{THz}$ porous wave guide with a flat dispersion from 0.98 to $1.15 \mathrm{THz}$ frequency range with absolute dispersion variation less than $2.5(\mathrm{ps} / \mathrm{THz} / \mathrm{cm})$. This design reported effective material loss was $0.432 \mathrm{~cm}^{-1}$. Last year, Shaopeng $\mathrm{Li}$ et al. [21] proposed a triangular-lattice, porous core fibre without a centre elliptical air hole in their design. They reported a low EML with nearly zero dispersion curves in the frequency range of 0.1 to $5 \mathrm{THz}$. Shubi Kaijage et al. proposed a porous $\mathrm{THz}$ waveguide using an octagonal air hole distribution with a central circular air hole [12]. The proposed design reported a low material absorption loss of $0.07 \mathrm{~cm}^{-1}$ at $1 \mathrm{THz}$ operating frequency. In 2014, Imran et al. proposed a circular microstructure core having four air hole rings with a central air hole using octagonal cladding in the design. This porous core waveguide resulted a low EML of $0.056 \mathrm{~cm}^{-1}$ and near zero flat dispersion with absolute dispersion variation of $\pm 0.18 \mathrm{ps} / \mathrm{THz} / \mathrm{cm}$ in the frequency range from 1.0 to $1.8 \mathrm{THz}$ [9]. Recently in 2015, Islam et al. proposed a low-loss rotated porous core hexagonal fiber using the same TOPAS material [22]. They have reported a low material absorption loss of $0.066 \mathrm{~cm}^{-1}$ and near zero flat dispersion of $1.06 \pm 0.12$ $\mathrm{ps} / \mathrm{THz} / \mathrm{cm}$ from $0.5-1.08 \mathrm{THz}$ frequency range [22].

In the reported design a hexagonal cladding has been used with a newly proposed hybrid core structure [1]. With this improved air hole arrangement the proposed fiber has shown 
very low EML with flat characteristics for $3.5 \mathrm{THz}$ frequency range. Negligible confinement and bending loss with large range of near zero flat dispersion has been achieved using this design. The hybrid core of the fiber has combined a circular and a triangular air hole distribution. In this paper we have discussed the impact on the waveguide properties for rotating the triangular air hole arrangement in the hybrid porous core. The triangular core has been rotated for $30^{\circ}, 60^{\circ}$ and $90^{\circ}$ from the optimum design and all effects on waveguide properties have been investigated and compared to each other. From the effects reported here we can observe a clear view of the internal physics and characteristics of the porous core $\mathrm{THz}$ waveguide.

\section{Design Methodology}

COMSOL Multiphysics (version 4.2) has been used to arrange the structure of the fiber. Finite element method (FEM) with a perfectly matched layer (PML) has been used to study the propagation characteristics of the waveguide. The cladding of the fiber has a hexagonal distribution with three air hole rings. Number of air holes in these rings is 24,30 and 36 respectively. The core region of the proposed fiber has a hybrid arrangement of air holes having six air holes in a triangular lattice design surrounded by two circular rings. Outer circular ring has 18 air holes which are $20^{\circ}$ apart from one another and the inner circular ring has 12 air holes in $30^{\circ}$ apart. All the air holes used in the design are circular shaped. A perfectly matched layer (PML) has been introduced surrounding the fiber body with $13 \%$ thickness of the fiber radius. In this paper we have rotated the triangular air hole arrangement by $30^{\circ}, 60^{\circ}$ and $90^{\circ}$ to investigate the effects while keeping other air holes while keeping the other air holes at the same place. Fig. 1 shows the design structure of the reported fiber for optimum parameters.

The core diameter of the porous fiber is denoted as Dcore; pitch (air hole to air hole distance) of the core and cladding are denoted as $\Lambda_{1}$ and $\Lambda_{2}$. All the air holes in the hybrid core has same radius $\left(r_{1}\right)$ and the radius of the cladding air holes is $r_{2}$. The values for optimum design parameters are as follows: Dcore $=2450 \mu \mathrm{m}, \Lambda_{1}=350 \mu \mathrm{m}, \Lambda_{2}=381 \mu \mathrm{m}, r_{1}=117.9 \mu \mathrm{m}$ and $r_{2}=171.4 \mu \mathrm{m}$. Due to the fact that, only circular air holes have been used in the fiber design and the ait hole arrangement is very simple; thus a capillary stacking fabrication process can be used for the practical fabrication of the reported design [9].

The host material used for the design is TOPAS; also chemically known as cyclic-olefin copolymer [23]. It has a constant refractive index of 1.5258 in the $\mathrm{THz}$ spectrum $(0.1$ 2.0 THz) and a low bulk material loss of $0.2 \mathrm{~cm}^{-1}$ at $1 \mathrm{THz}$ frequency [24]. Such properties are very beneficial for resulting in near zero flat dispersion [24] and low EML. Also the material does not absorb water [25], it is insensitivity to humidity [25] and it has chemical inertness with special biosensing properties [26].

\section{RESULT \& DISCUSSION}

The triangular air holes in the hybrid core region are rotated in anti-clockwise direction for $30^{\circ}, 60^{\circ}$ and $90^{\circ}$ and simulation results are plotted for different waveguide properties. In fig. 2 , we can see the structural change in the design for rotating the triangular core. Here it can be seen that, the triangular core geometry for optimum (Fig. 2.a) and $60^{\circ}$ rotation (Fig. 2.c) are asymmetric with respect to $\mathrm{x}$-axis. Due to the other parameters are similar for these two designs, they have appeared vertically flipped. Thus their optical properties and results should be similar except the resulted fundamental modes for transverse electric (TE) field will be interchanged. But the optimum design of the reported fiber has a very little asymmetry in the design (Fig. 1). So the optical properties for $\mathrm{x}$ and $\mathrm{y}$ polarizing signals have very similar outputs. Thus, even with resulting in opposite polarizations, these two designs will almost serve close results. Similar phenomena will occur with $30^{\circ}$ (Fig. 2.b) and $90^{\circ}$ (Fig. 2.d) rotations as they have appeared to be horizontally flipped designs of each other. We have investigated this theory and discuss the results in this paper for different waveguide properties. COMSOL Multiphysics

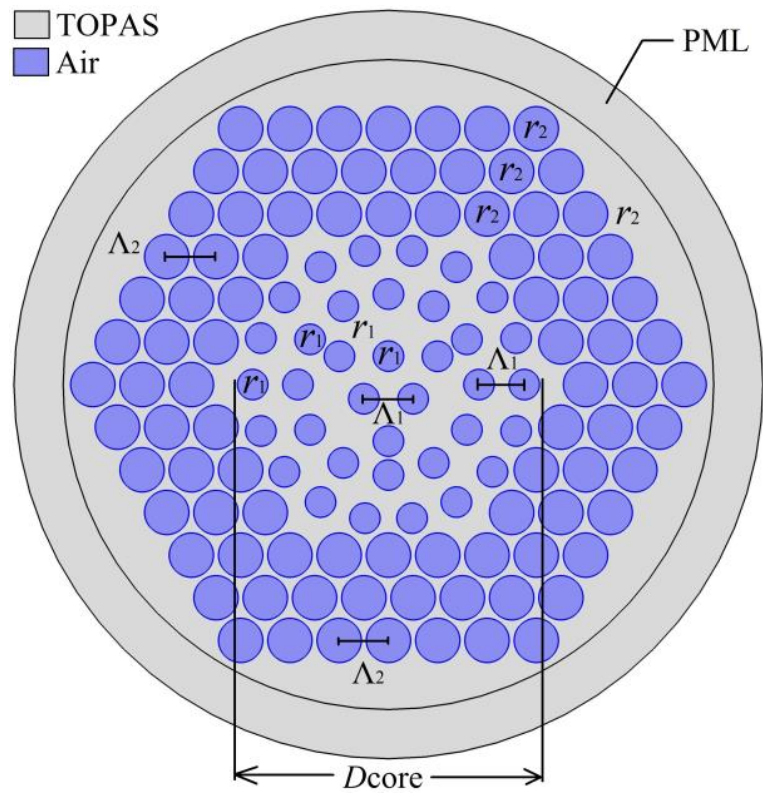

Fig. 1. Air hole arrangement of the reported hybrid porous core fiber.

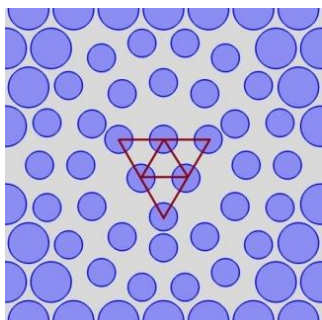

(a)

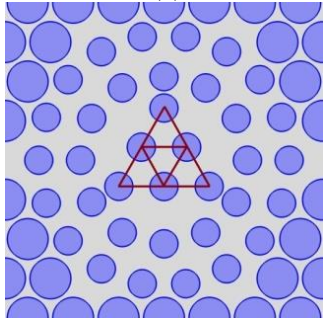

(c)

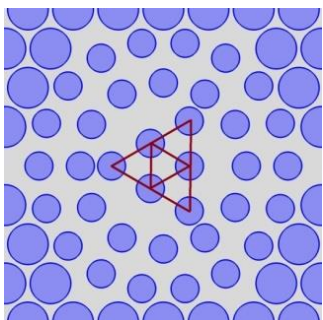

(b)

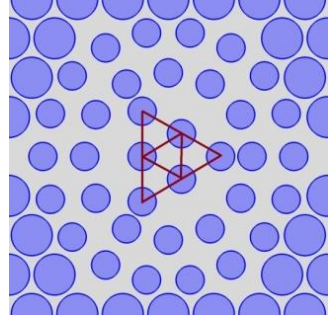

(d)
Fig. 2. Design structure of air hole arrangement for rotating the triangular core in anti-clockwise direction (a) optimum design $\left(0^{\circ}\right.$ rotation), (b) $30^{\circ}$ rotation, (c) $60^{\circ}$ rotation, (d) $90^{\circ}$ rotation. 
simulator has been used to determine the single mode confinements for the transmitted light and different waveguide properties for those confinements are investigated; such as: effective refractive index, material loss, power fraction etc. All the results are then plotted in MATLAB using designated equations to observe the changes graphically.

Fig. 3 and fig. 4 are showing the fundamental mode filed distribution for both $\mathrm{x}$ and $\mathrm{y}$ polarizations respectively of transverse electric (TE) field for single mode confinements of different rotations. Arrow directions are showing the polarization direction of the transverse electric fields. All the fundamental mode filed confinements have been simulated for $1 \mathrm{THz}$ operating frequency. It can be observed that the difference between the modes for $\mathrm{x}$ and $\mathrm{y}$ polarizations of each rotation are very similar due to the low amount of asymmetry in the hybrid porous core design. The field distributions for optimum to $60^{\circ}$ rotation and $30^{\circ}$ rotation to $90^{\circ}$ rotation can be considered just flipped vertically and horizontally respectively. We have discussed the physics behind this phenomenon in the above paragraph. The changes in the mode field distributions have followed the theory we have discussed before.

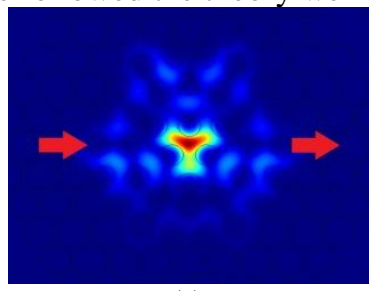

(a)

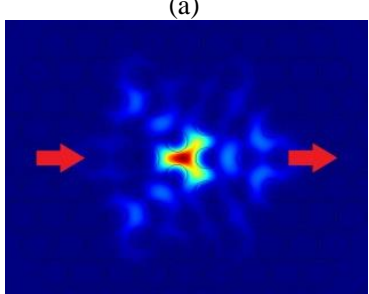

(c)

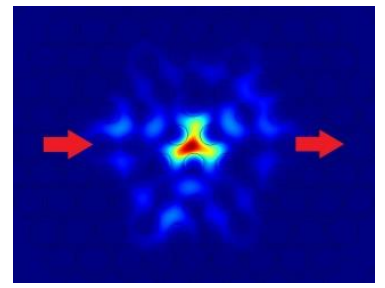

(b)

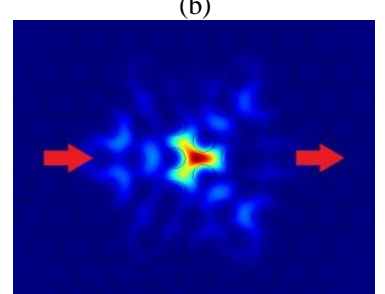

(d)

Fig. 3. Fundamental mode filed distribution for $\mathrm{x}$ polarization of (a) optimum design position, (b) $30^{\circ}$ rotation, (c) $60^{\circ}$ rotation and (d) $90^{\circ}$ rotation.

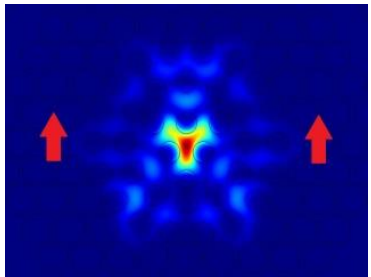

(a)

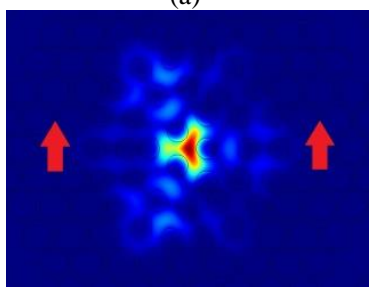

(c)

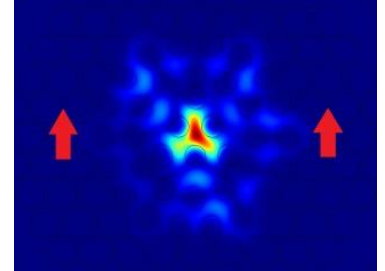

(b)

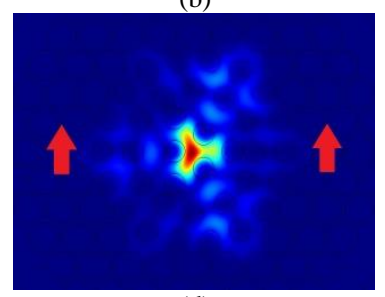

(d)
Fig. 4. Fundamental mode filed distribution for y polarization of (a) optimum design position, (b) $30^{\circ}$ rotation, (c) $60^{\circ}$ rotation and (d) $90^{\circ}$ rotation.

Now we investigate the effective refractive index of the reported fiber. As discussed before the single mode confinements are almost similar for the $\mathrm{x}$ and $\mathrm{y}$ axis of all rotation designs, thus their effective indexes of each polarization will be same. So we have only represented the $x$ axis polarization effective index in fig. 5(a). It can be seen that all the refractive indexes for different rotation are overlapping one another. This is due to the similarity of the design even after the triangular core has been rotated up to $90^{\circ}$. We can also see that the effective indexes increase with increasing frequency and after a certain point it has an almost flat value close to the refractive index of the used host material TOPAS (i.e., 1.5258). The reason behind this is the transmitting mechanism of light inside the fiber. The porous design fiber confines traveling light using total internal reflection (TIR) system. In this system light waves get reflected while travelling from an optically denser medium to a lower denser medium when the incident angle of the wave is larger than the critical angle for that medium. In the reported hybrid porous core design the centre point of the core is surrounded by triangular air holes witch has created the perfect scenario for TIR. Thus light is tightly confined in that central core point. As a result the effective index has got less diverted by the array of air holes in the rest of the core and cladding. So the resulted effective index has got close to the refractive index of the material and with the increased frequency resembled closer values to each other. In the end the effective index of the porous fiber showed an almost flat value close to the refractive index of the material. The credit for this interesting phenomenon goes to the creative design of the fiber. Individual fiber designs will obviously show different characteristics due to their own design architectures.

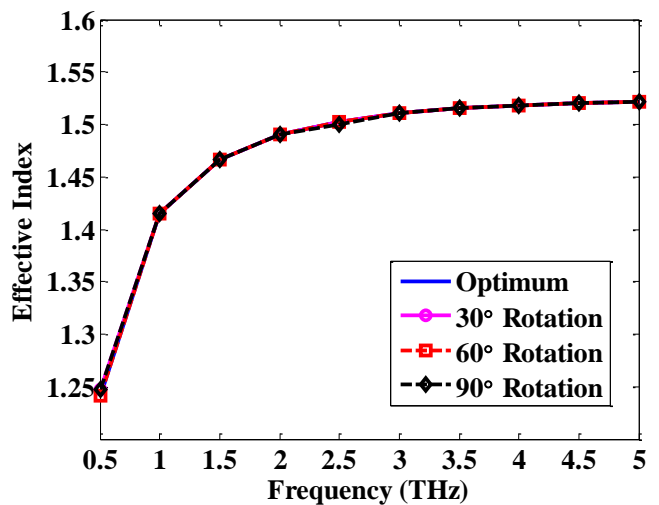

(a)

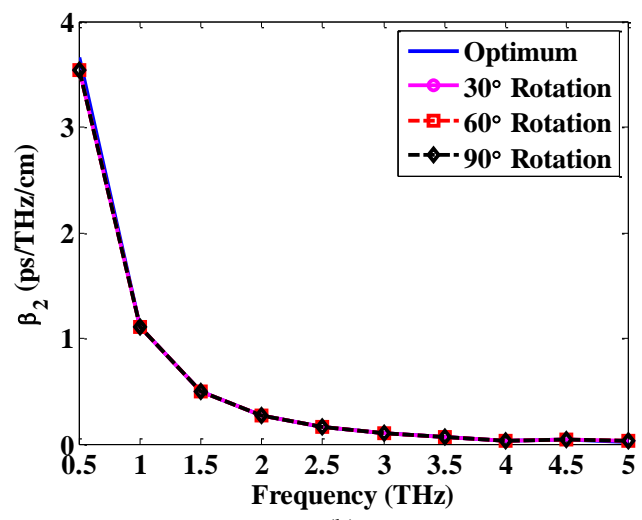

(b)

Fig. 5. (a) Effective refractive index of the reported hybrid core fiber and (b) dispersion of the reported design. 
The dispersion of the group velocity of the reported hybrid porous core design can be represented with respect to the frequency by calculating the coefficient $\beta_{2}(\mathrm{ps} / \mathrm{THz} / \mathrm{cm})$. This term corresponds to the second order term in the Taylor expansion of the modal propagation constant $\beta$. The equation can be expressed in the following manner where, $\omega=2 f \pi$, and $n_{\mathrm{eff}}=\operatorname{Re}(\beta) c / \omega$, constant $c$ is the velocity of light [9].

$$
\beta_{2}=\frac{2}{c} \frac{d n_{e f f}}{d \omega}+\frac{\omega}{c} \frac{d^{2} n_{e f f}}{d \omega^{2}}
$$

We can see from the equation that the value of $\beta_{2}$ in $(\mathrm{ps} / \mathrm{THz} / \mathrm{cm})$ is directly affected by the effective refractive index of the profile. Thus due to the flatness of the effective index the dispersion characteristics will also have large flatness as a function of frequency. Dispersion characteristics of all rotation designs have been evaluated and represented in fig. 5(b). According to the dissuasion before we can see that the curves for different rotation designs have overlapped each other and resulted in a very large near zero flat dispersion. The resulted near zero flat dispersion is $0.4 \pm 0.042 \mathrm{ps} / \mathrm{THz} / \mathrm{cm}$ in the frequency range of 1.25 to $5.0 \mathrm{THz}$. This is about $3.75 \mathrm{THz}$ band of flat dispersion with very small amount of absolute dispersion coefficient.

The EML of the fiber can be determined by using the following equation [20];

$$
\alpha_{e f f}=\frac{\alpha_{m o}}{\alpha_{\text {mat }}}=\frac{1}{2}\left(\frac{\varepsilon_{0}}{\mu_{0}}\right)^{1 / 2} \frac{\int n_{\text {mat }}|E|^{2} d A}{\left|\int_{\text {all }} S_{z} d A\right|}
$$

Here $\alpha_{\text {eff }}$ is representing the EML of the fiber, $\alpha_{m o}$ is the loss of fundamental mode, $\alpha_{\text {mat }}$ is the loss from material absorption, $\varepsilon_{0}$ is the permittivity and $\mu_{0}$ is the permeability of free space [9]. The refractive index $n_{\text {mat }}$ is calculated by the simulator. $s_{z}$ is pointing vector of $z$ - component where $s_{z}=1 / 2 \vec{E} \times \overrightarrow{H . z}$ ( $E$ is the electric, $H$ is the magnetic field). We have brought down several investigations regarding EML. At first the changes in EML for triangular core rotation for different core diameters has been presented in fig. 6(a). All values have been taken for optimum porosity of $33.32 \%$ and $1 \mathrm{THz}$ operating frequency. For having a flipped design structure of optimum with $60^{\circ}$ rotations and $30^{\circ}$ rotations with $90^{\circ}$ rotations as discussed earlier, a similarity of EML can be observed among them. Also it can be seen that with the larger value of Dcore the EML differences for different rotations have drawn smaller. Larger core diameter causes light waves exposed more area of the material and resulted in a higher EML.

We have investigated the EML values for different porosity at optimum core diameter $2450 \mu \mathrm{m}$ and $1 \mathrm{THz}$ frequency. The effect on different triangular core rotation designs for the change of porosity is vital. Usually for higher porosity the transmitted light has a better chance to radiate through the air holes causing lower EML. The similar thing also happened in the reported design. Due to the confinement structure is little different than others for different core rotation designs showed in fig. 3 and fig. 4 , the amount of light travelling through air holes is also different for those rotations. Thus the resulted
EML has large dissimilarities for different rotation designs at changed porosity. This incident can be well observed from the fig. 6(b) where several points have shown very different EML values. At a particular porosity for a certain design if the value of the EML is lower than other that means at that design parameters, the travelling light has radiated less through the air holes and more through the material. The phenomenon is just the opposite for a higher EML value at a particular point of a design.

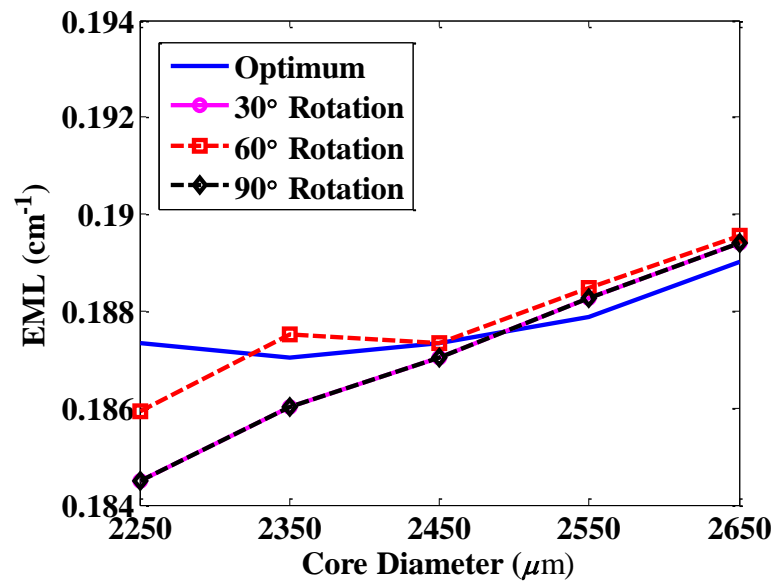

(a)

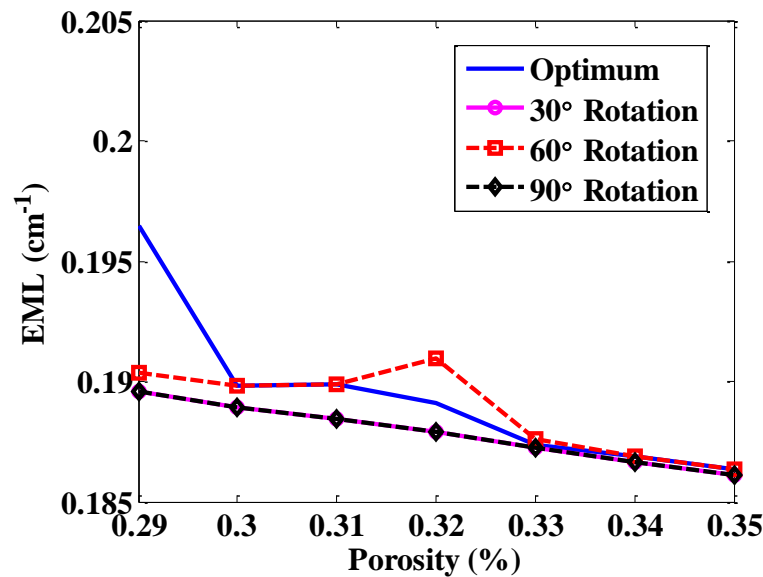

(b)

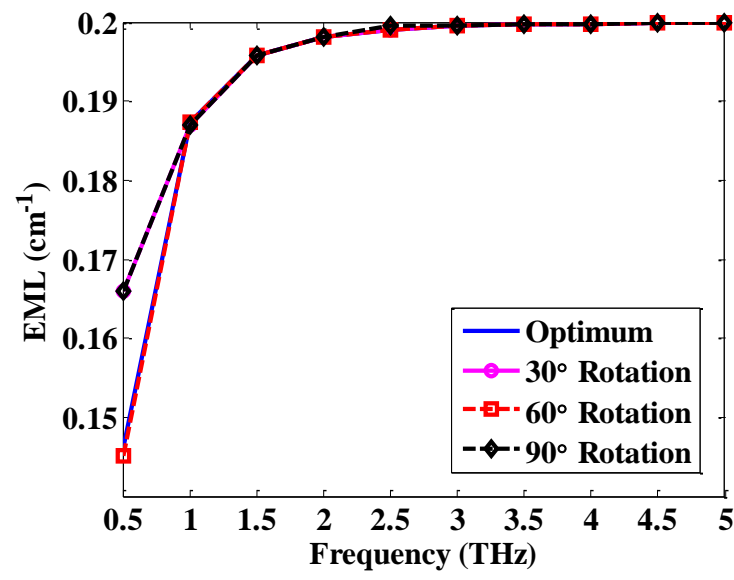

(c)

Fig. 6. EML at $1 \mathrm{THz}$ frequency in (a) different core diameters for $33.32 \%$ porosity, (b) for different porosity for $2450 \mu \mathrm{m}$ core diameter and (c) EML for optimum parameter of $2450 \mu \mathrm{m}$ core diameter and $33.32 \%$ porosity. 
The EML for optimum parameters of $2450 \mu \mathrm{m}$ core diameter and $33.32 \%$ porosity has been exhibited in fig. $6(\mathrm{c})$. For the design similarities in optimum and $60^{\circ}$ rotations and in $30^{\circ}$ and $90^{\circ}$ rotations discussed before, the EML has been resulted similar. For higher frequency values all rotation designs have shown almost same EMLs. In case of lower frequency (< $1 \mathrm{THz}$ ) a change can be seen in the EML results. For lower frequency the effective index changed rapidly and changed the confinement property of the propagation. As a result the power flow also changed for that and thus the EML value of the reported fiber. Most of the changes in the power flow happened in the cladding air holes and in the material. In the following paragraph we have discussed about this effect.

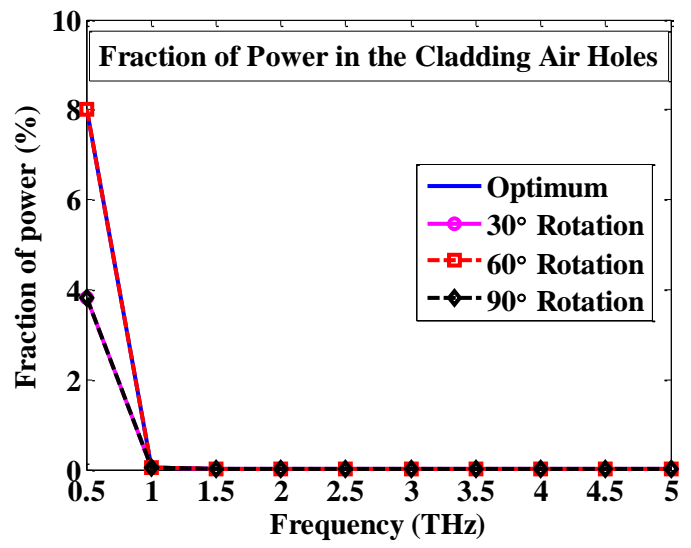

(a)

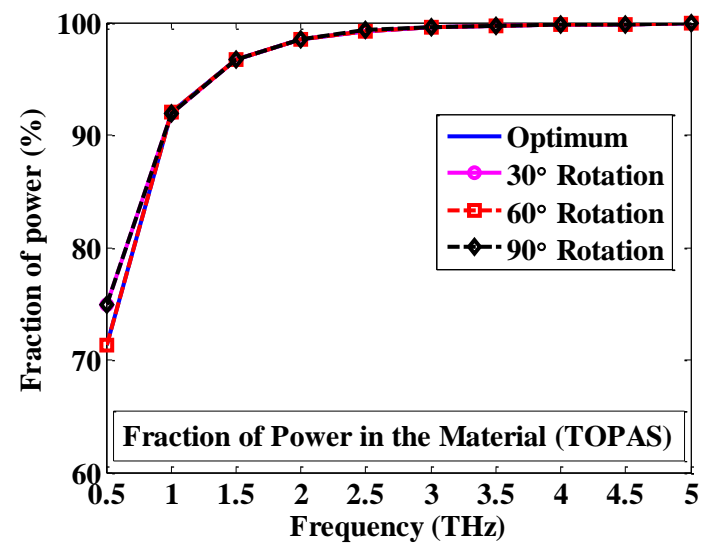

(b)

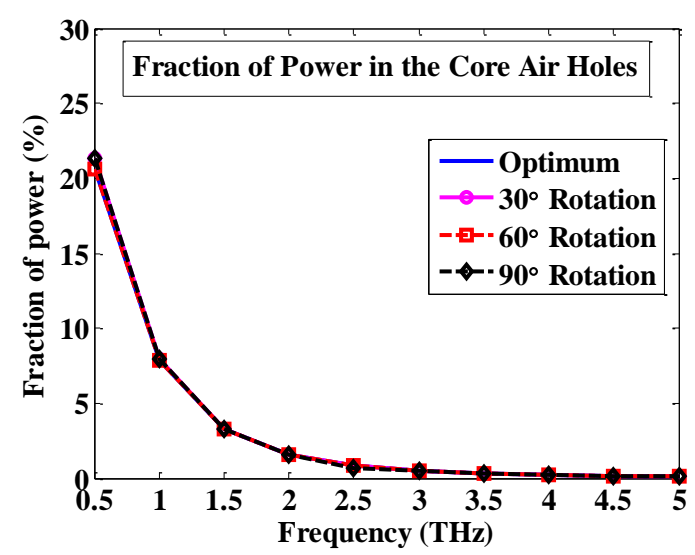

(c)

Fig. 7. Fraction of power as a function of frequency (a) in the cladding air holes, (b) in the material (TOPAS) and (c) in the core air holes.
In fig. 7(a) we can observe the changes in the power flow in the cladding air holes for different rotation designs of the triangular core. For optimum and $60^{\circ}$ rotation the amount of power transmitted through the cladding air holes has larger percentage than the other two rotation designs under $1 \mathrm{THz}$ frequency. On the other hand, the power flow in the material has reduced value under $1 \mathrm{THz}$ frequency for optimum and $60^{\circ}$ rotation designs which can be seen at fig. 7(b). Although the power flow in the core air holes has remained same for all the different rotation designs shown in fig. 7(c); it can be observed from these three figures that the overall power flow in the material has decreased for optimum and $60^{\circ}$ rotation designs for frequency lower than $1 \mathrm{THz}$. If the amount of power flowing is low inside the material so as the value of EML for that particular frequency. Judging from fig. $7,30^{\circ}$ and $90^{\circ}$ rotation designs have experienced a less percentage of power flow through the air holes and more power has been transmitted inside the material under $1 \mathrm{THz}$ frequency. Thus they showed a higher EML under $1 \mathrm{THz}$ frequency as it has been discussed in fig. 6(c).

Although the changes occurred for rotating the triangular core has affected the power flow in the material and cladding air holes, the power flow inside the core air holes has been similar for all rotation designs as illustrated in fig. 7(c). The amount of power has decreased in the core air holes with higher frequency due to the effect of compact single mode confinements in the central point of the core. This is due to the increased effective refractive index with increasing frequency which has been discussed in fig. 5(a). For the TIR mechanism of transmitted light with higher effective index the confinements are tight in the central point of the core and less affected by the air holes and surrounding materials in the cladding. As a result the power flow has decreased in the core air holes with increased frequency. With this very tight confinement the propagating light experienced less impact from the whole materials and consumed by only a small portion of material in the core region. This resulted in a low EML for the reported hybrid porous core fiber.

Usually porous core fibers have three types of considerable losses; such as effective material loss (EML), confinement loss and bending loss. We have already discussed about EML previously in this paper. The effect on confinement loss has been shown in fig. 8(a) for different rotations. The confinement loss, $\alpha_{C L}$ can be expressed by the following equation [9] where $f$ is the frequency of the light, $c$ is the velocity of the light in vacuum (constant) and $\operatorname{Im}\left(n_{\text {eff }}\right)$ is the imaginary part of the refractive index of the guided mode which has been derived by the simulator;

$$
\alpha_{C L}=8.686 \frac{2 \pi f}{c} \operatorname{Im}\left(n_{e f f}\right)
$$

We can see some changes in the confinement loss for certain frequencies of different rotation designs. This happened for the change in the core air hole arrangements for rotating the triangular core. The confinement loss depends on the cladding and core air hole position to guide the propagation towards a particular confinement. Whenever the balance has been changed between the core and cladding air holes the confinement loss also changes. Similar phenomenon can be 
observed for bending loss plotted in fig. 8(b). A recently reported conformal transformation method [22] has been used to calculate the bending loss with a modified index profile. The following equation represents the modified equivalent index for bending loss of the fiber.

$$
n_{e q}(x, y)=n(x, y)\left(1+\frac{x}{R}\right)
$$

Here $n_{\mathrm{eq}}(x, y)$ is the modified equivalent index distribution, $n(x, y)$ is the original refractive index profile of the PCF, $R$ is the bending radius (bending radii $=1 \mathrm{~cm}$ ) and $x$ is the distance from the centre of the fiber to the bending point. Once the $n_{\text {eq }}(x, y)$ is calculated, bending loss can be found by using the same formula as described for determining confinement loss.

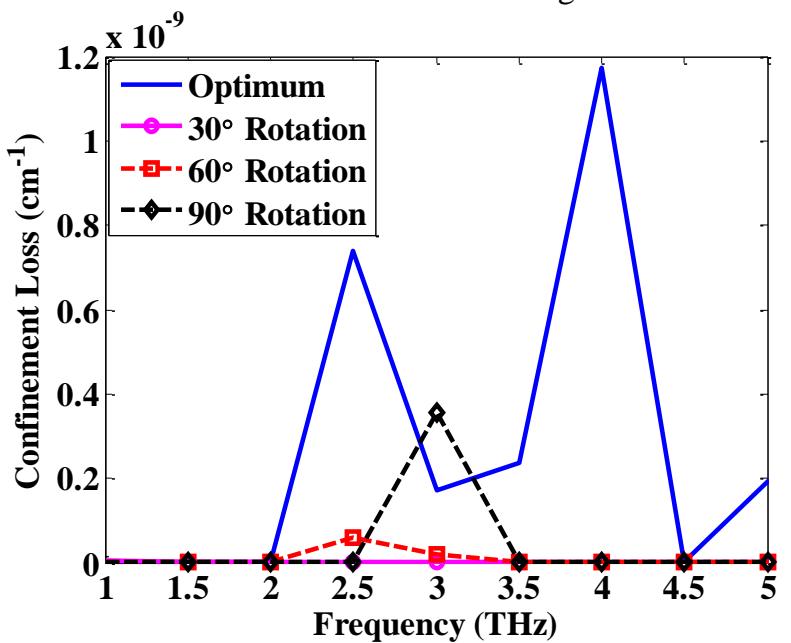

(a)

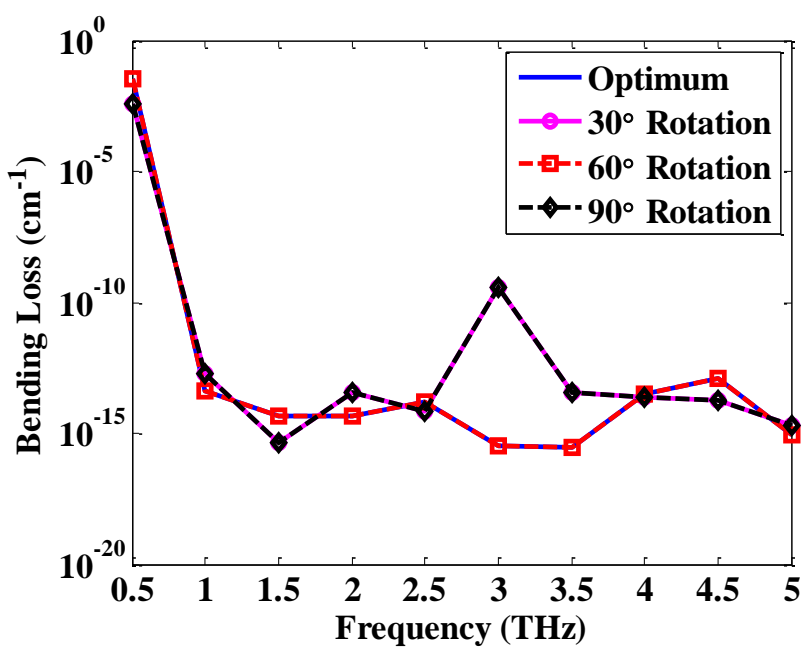

(b)

Fig. 8. (a) Confinement loss and (b) bending loss as a function of frequency.

Similar to the physics of the changes occurred in confinement loss, bending loss also showed some changes for different triangular core arrangements. Although the value of bending radii $R$ and distance from the centre of the fiber to the bending point $x$ has been same for all rotation designs, the different confinement loss for changing the core and cladding air hole balance has caused the changes in the bending loss for different rotation designs.

\section{CONCLUSION}

We have investigated the impact of changing the triangular core position on different waveguide properties by rotating the triangular core. We have reported the effect of air hole arrangements on effective refractive index and thus resulting in a large range (3.75 $\mathrm{THz}$ band) near zero flat dispersion at $0.4 \pm 0.042 \mathrm{ps} / \mathrm{THz} / \mathrm{cm}$. The physics behind the low flat EML of $\pm 0.000416 \mathrm{~cm}^{-1}$ from 1.5 to $5 \mathrm{THz}$ for the reported design has been discussed. The changes in power fraction affecting the EML for rotating the triangular core have been discussed. The cause and effect of rotating triangular air holes on confinement and bending loss has been reported. We have learned from these investigations that the changing of air hole positions by rotating the core can bring some interesting effects on waveguide characteristics. If the design has lower asymmetry then similar looking rotations having a vertical or horizontal flipped position of air holes can have almost same waveguide properties. Using this study other porous core designs can be tuned for determining more possible variations in their waveguide properties.

\section{REFERENCES}

[1] Sharafat Ali, Nasim Ahmed, Syed Aljunid, Badlishah Ahmad, "Ultra-flat low material loss porous core $\mathrm{THz}$ waveguide with near zero flat dispersion," Electronics Letters, vol. 52, pp. 863-865, 2016.

[2] Y. F. He, P.I. Ku, J.R. Knab, J.Y. Chen, A.G. Markelz, "Protein Dynamical Transition Does Not Require Protein Structure," Physical Review Letters, vol. 101, p. 178103, 2008.

[3] J. Q. Zhang, D. Grischkowsky, "Waveguide terahertz time-domain spectroscopy of nanometer water layers," Optics Letters, vol. 29, pp. 1617-1619, 2004.

[4] L. Ho, M. Pepper, P. Taday, "Terahertz spectroscopy: Signatures and fingerprints," Nature Photonics, vol. 2, pp. 541, 2008.

[5] C. J. Strachan, P.F. Taday, D.A. Newnham, K.C. Gordon, J.A. Zeitler, M. Pepper, T. Rades, "Using Terahertz Pulsed Spectroscopy to Quantify Pharmaceutical Polymorphism and Crystallinity," Journal of Pharmaceutical Science, vol. 94, pp. 837-846, 2005.

[6] D. J. Cook, B.K. Decker, M.G. Allen, "Quantitative THz Spectroscopy of Explosive Materials," in OSA Conference, USA, 2005, pp. PSI-SR1196.

[7] Q. Chen, Z.P. Jiang, G.X. Xu, X.C. Zhang, "Near-field terahertz imaging with a dynamic aperture," Optics Letters, vol. 25, pp. 1122-1124, 2000.

[8] M. Nagel, Bolivar, P. H., Brucherseifer, M., Kurz, H., Bosserhoff, A., \& Büttner, R., "Integrated $\mathrm{THz}$ technology for label-free genetic diagnostics," Applied Physics Letters, vol. 80, pp. 154-156, 2002.

[9] M. I. Hasan, S. M. Abdur Razzak, G. K. M. Hasanuzzaman and Md Samiul Habib, "Ultra-Low Material Loss and Dispersion Flattened Fiber for THz Transmission," IEEE Photonic Technology Letters, vol. 26, pp 2372-2375, 2014.

[10] Na-na Chen, Jian Liang, and Li-yong Ren, "High-birefringence, low-loss porous fiber for single mode terahertz wave guidance," Applied Optics, vol. 52, pp. 5297-5302, 2013.

[11] M. Uthman, B. M. A. Rahman, N. Kejalakshmy, A. Agrawal, K. T. V. Grattan, "Design and Characterization of Low-Loss Porous-Core Photonic Crystal Fiber," IEEE Photonic Journal, vol. 4, pp. 2315-2325, 2012.

[12] S. Kaijage, Zhengbiao Ouyang, Xin Jin, "Porous-Core Photonic Crystal Fiber for Low Loss Terahertz Wave Guiding," IEEE Photonic Technology Letters, vol. 25, pp. 1454-1457, 2013.

[13] T. Jeon, J. Zhang, and D. Grischkowsky, "THz Sommerfeld wave propagation on a single metal wire," Applied Physics Letters, vol. 86, pp. 161704-1-161904-3, 2005.

[14] B. Bowden, J. A. Harrington, and O. Mitrofanov, "Silver/polystyrenecoated hollow glass waveguides for the transmission of terahertz radiation," Optic Letters, vol. 32, pp. 2945-2947, 2007.

[15] A. Dupuis, K. Stoeffler, B. Ung, C. Dubois, and M. Skorobogatiy, "Transmission measurements of hollow-core THz Bragg fibers," Journal of Optical Society of America, vol. 28, pp. 896-907, 2011. 
[16] M. Nagel, A. Marchewka, and H. Kurz, "Low-index discontinuity terahertz waveguides," Optic Express, vol. 14, pp. 9944-9954, 2006.

[17] K. Nielsen, Rasmussen, H., Peter Uhd Jepsen, Ole Bang, "Porous-core honeycomb bandgap THz fiber," Optic Letters, vol. 36, pp. 666-668, 2011.

[18] B. Hualong, Kristian Nielsen, Henrik K. Rasmussen, Peter Uhd Jepsen and Ole Bang, "Fabrication and characterization of porous-core honeycomb bandgap THz fibers," Optic Express, vol. 20, pp. $29507-$ 29517, 2012.

[19] S. Atakaramians, S. Afshar, M. Nagel, H.K. Rasmussen, O. Bang, T.M Monro and D. Abbott, "Direct probing of evanescent field for characterization of porous terahertz fibers," applied Physics Letters, vol 98, pp. 121104, 2011.

[20] J. Liang, Liyong Ren, Nana Chen, Changhe Zhou, "Broadband, low-loss, dispersion flattened porous core photonic band gap fiber for terahertz (THz) wave propagation," Optics Communications, vol. 295, pp. $257-$ 261, 2013.

[21] L. Shaopeng, Hongjun Liu, Nan Huang and Qibing Sun, "Broadband high birefringence and low dispersion terahertz photonic crystal fiber," Journal of Optics, vol. 16, pp. 105102, 2014.
[22] R. Islam, Hasanuzzaman GKM, Habib Selim, Rana Sohel, Khan MAG, "Low-loss rotated porous core hexagonal single-mode fiber in $\mathrm{THz}$ regime," Optical Fiber Technology, vol. 24, pp. 38-43, 2015.

[23] S. Rana, Sharafat Ali, Nasim Ahmed, Raonaqul Islam, Syed A. Aljunid, "Ultra-High Birefringent and Dispersion-Flattened Low Loss SingleMode Terahertz Wave Guiding," IET Communications, DOI:10.1049/ietcom.2015.0629, 2016.

[24] K. Nielsen, H. K. Rasmussen, A. J. L. Adam, P. C. M. Planken, O. Bang and P. U. Jepsen, "Bendable, low-loss Topas fibers for the terahertz frequency range," Optic Express, vol. 17, pp. 8592-8601, 2009.

[25] G. Emiliyanov, J. Jensen, O. Bang, P. Hoiby, L. Pedersen, E. Kjær, and L. Lindvold, "Localized biosensing with Topas microstructured polymer optical fiber," Optic Letters, vol. 32, pp. 460-462, 2007.

[26] G. Emiliyanov, Poul E. Høiby, Lars H. Pedersen and Ole Bang, "Selective serial multi-antibody biosensing withTOPAS microstructured polymer optical fibers," Sensors, vol. 13, pp. 3242-3251, 2013. 